\title{
ON CONVEX TOPOLOGICAL LINEAR SPACES $\left({ }^{1}\right)$
}

BY

\author{
GEORGE W. MACKEY
}

Introduction. In an earlier article [9] the author developed at some length the theory of certain mathematical objects which he called linear systems. It is the purpose of the present paper to apply this theory to the study of convex topological linear spaces. This application is based on the many-to-one correspondence between convex topological linear spaces and linear systems which may be set up by assigning to each such space $X_{t}$ the linear system $X_{L}$ where $X$ is the abstract linear space underlying $X_{t}$ and $L$ is the set of all continuous linear functionals on $X$. First of all the nature of the family of all $X_{t}$ 's belonging to a given $X_{L}$ is studied and it is shown that it has a weakest and a strongest member. The bulk of the paper is then devoted to correlating the properties of a convex topological linear space with those of its linear system and with the strength of its topology relative to that of the others with the same linear system. A general survey of the contents of this paper will be found in [10].

1. Preliminary definitions and remarks. In-this section we recall briefly some of the notions from the theory of topological linear spaces and from [9] which we shall need in the sequel. By a topological linear space $\left({ }^{2}\right)$ we mean a real linear space which is at the same time a $T_{1}$ space in the sense of Alexandroff and Hopf [1] and in which the topology is related to the algebra in such a manner that the operations of addition and multiplication by reals are continuous in both variables together. By a convex topological linear space $\left({ }^{2}\right)$ we mean a topological linear space in which every point has a complete system of convex neighborhoods. By a linear functional on a linear space we mean a function $l$ defined from the space to the reals such that $l(\lambda x+\mu y)=\lambda l(x)$ $+\mu l(y)$ for all $x$ and $y$ in the space and all real numbers $\lambda$ and $\mu$. If $X$ is a. linear space we denote by $X^{*}$ the linear space of all linear functionals on $X$. By a linear system $X_{L}$ we mean a linear space $X$ together with a distinguished

Presented to the Society, April 27, 1946; received by the editors March 13, 1946.

(1) Most of the results of this paper, like those of its predecessor, have been drawn from the author's doctoral thesis, Harvard 1942. Some of the work on the latter was done while the author was a Sheldon travelling fellow from Harvard University in residence at the California Institute of Technology and the Institute for Advanced Study. The author wishes to acknowledge his indebtedness to Professor M. H. Stone at whose suggestion the part of the thesis containing the results of this paper was written. Numbers in brackets refer to the bibliography at the end of the paper.

(2) These notions have been introduced in slightly different ways by various authors. See Wehausen [18] for a discussion. See also footnote 22 of Whitney [20] for a discussion of a popular misconception. 
subspace $L$ of $X^{*}$. If $L$ is total in the sense that $l(x)=0$ for all $l$ in $L$ implies that $x=0$ we say that $X_{L}$ is regular. The linear system $L_{F}$ where $F$ is the subset of $L^{*}$ consisting of those functionals defined by members of $X$ is called the conjugate of $X_{L}$ and is denoted by $X_{L}^{0}$ or by $L_{X}$. A subset $A$ of $X_{L}$ is said to be bounded if for each countable sequence of elements of $A, x_{1}, x_{2}, \cdots$, and each $l$ in $L$ the sequence of real numbers $\left\{l\left(x_{n}\right)\right\}$ is a bounded sequence. If $l$ is a member of $X^{*}$ such that $\left\{l\left(x_{n}\right)\right\}$ is a bounded sequence of real numbers whenever $\left\{x_{n}\right\}$ is a bounded sequence of members of $X_{L}$ then $l$ is said to be in the bounded closure of $L$. If each such $l$ is in $L$ then $L$ and $X_{L}$ are said be boundedly closed. If $\left\{l_{n}\left(x_{m}\right)\right\}$ is bounded for $n, m=1,2, \cdots$ whenever $\left\{l_{n}\right\}$ is a bounded subset of $L_{X}$ and $\left\{x_{m}\right\}$ is a subset of $A \subseteq X$ we say that $A$ is a uniformly bounded subset of $X_{L}$. If every bounded subset of $X_{L}$ is uniformly bounded we say that $X_{L}$ is uniform. If there exists a bounded subset of $X_{L}$ whose linear span is $X$ we say that $L$ and $X_{L}$ are relatively bounded. If $X$ is the union of countably many bounded subsets of $X_{L}$ we say that $L$ and $X_{L}$ are almost relatively bounded. Let $x_{1}, x_{2}, \ldots$ be a sequence of members of $X$. If there exists a member $f$ of $L^{*}$ and a sequence $\gamma_{1}, \gamma_{2}, \cdots$ of positive real numbers converging to zero such that $\left\{\left(l\left(x_{n}\right)-f(l)\right) / \gamma_{n}\right\}$ is bounded for all $l$ in $L$ we say that $x_{n}$ converges to $f$ in the linear system $X_{L}$. If whenever $x_{n}$ converges to $f$ there exists $\bar{x}$ in $X$ such that $f(l)=l(\bar{x})$ for all $l$ in $L$ we say that $X_{L}$ is a complete linear system. A function $N$ from a linear space $X$ to the non-negative reals such that $N(x+y) \leqq N(x)+N(y)$ and $N(\lambda x)=|\lambda| N(x)$ for all $x$ and $y$ in $X$ and all real numbers $\lambda$ is what we call a pseudo-norm. If $N(x)=0$ implies that $x=0$ we call $N$ a norm. If $N$ is a pseudo-norm, the pseudo-norm set of $N$ is the set of all $l$ in $X^{*}$ such that, for some positive real number $\mu$ depending on $l$, the inequality $|l(x)| \leqq \mu N(x)$ holds for all $x$ in $X$. It is a trivial generalization of a theorem of Fichtenholz [5] that the pseudonorm set of $N_{1}$ includes that of $N_{2}$ if and only if there exists a positive real number $\mu$ such that $N_{2}(x) \leqq \mu N_{1}(x)$ for all $x$ in $X$. A linear system $X_{L}$ is simple if the bounded closure of $L$ is a pseudo-norm set or, equivalently, if there exists a bounded subset of $X_{L}$ whose linear span is the whole space. If $A$ is a bounded subset of the conjugate of the linear system $X_{L}$ then l.u.b. $l_{\in A}|l(x)|$ is a pseudo-norm as a function of $x$. The linear span $L_{r}$ of the pseudo-norm sets of all such pseudo-norms is the reach of $L$ and $X_{L r}$ is the reach of $X_{L}$.

2. The convex topologies associated with a fixed linear system. Our theory of the family of convex topologies associated with a fixed linear system is based upon von Neumann's [12] observation that a convex topology may be described by pseudo-norms. Let $X$ be a convex topological linear space and let $O^{\prime}$ be any open set containing the origin. Then there exists a second open set $O$ which in addition to containing the origin and being contained in $O^{\prime}$ is convex and such that $-O=O$. For each $x$ in $X$ let $N(x)=1$.u.b. $|\lambda|$ for $x / \lambda \notin O$. It is readily verified that $N$ is a pseudo-norm and that $O$ is the set of all $x$ in $X$ with $N(x)<1$. Furthermore $N$ is continuous. In fact $N$ is obvi- 
ously continuous at the origin and it follows from the inequality $|M(x)-M(y)|$ $\leqq M(x-y)$ that any pseudo-norm $M$ is continuous if and only if it is continuous at the origin. Thus the sets (all $x$ with $N(x)<1$ ) for the continuous pseudonorms $N$ form a complete system of neighborhoods of the origin. The truth of the following theorem is an immediate consequence.

Theorem 1. Let $X$ be a linear space. Then two convex topologies in $X$ are identical if and only if the same pseudo-norms are continuous with respect to each.

According to Theorem 1 there is a natural one-to-one correspondence between the convex topologies in $X$ and certain families of pseudo-norms. Theorem 2 tells us just what these certain families are.

Theorem 2. A family $\mathfrak{R}$ of pseudo-norms $N_{\alpha}$ on a linear space $X$ is the family of all pseudo-norms continuous with respect to some convex topology $t$ in $X$ if and only if the following conditions are satisfied.

(a) If $N_{1} \in \mathfrak{N}$ and $N_{2}$ is such that $N_{2}(x) \leqq \mu N_{1}(x)$ for all $x$ in $X$ and some positive $\mu$ independent of $x$ then $N_{2}$ is also in $\Re$.

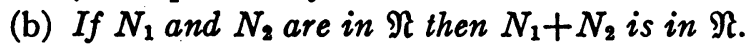

(c) If $x \in X$ and $x \neq 0$ then there exists $N$ in $\mathfrak{R}$ with $N(x) \neq 0$.

Proof. To prove the "only if" part of the theorem we observe that if $\mathfrak{N}$ is the family of continuous pseudo-norms for a convex topology in $X$ then condition (c) follows from the fact that the sets (all $x$ with $N(x)<1$ ) for the $N$ in $\mathfrak{N}$ form a complete system of neighborhoods of the origin and that condition (b) is a consequence of the continuity of the sum of two continuous: functions. (It is clear of course that the sum of two pseudo-norms is always a pseudo-norm.) Condition (a) follows from the fact that if $N_{1}$ is a continuous pseudo-norm and $N_{2}(x) \leqq N_{1}(x)$ for all $x$ in $X$ then the set where $N_{2}(x)<1$ contains the open set on which $N_{1}(x)<1$. Turning to the converse supposethat $\mathfrak{N}$ is a family of pseudo-norm sets satisfying (a), (b), and (c). Define a set $S$ in $X$ to be open if and only if for each $x$ in $X$ there is an $N$ in $\mathfrak{R}$ such that $N(x-y)<1$ implies that $y$ is in $S$. Then it may be verified without diffculty that with these open sets $X$ becomes a convex topological linear space. Let $N_{1}$ be any pseudo-norm continuous with respect to this topology. Then the set where $N_{1}(x)<1$ is open. Hence there exists $N_{2}$ in $\mathfrak{N}$ such that $N_{2}(x)<1$ implies that $N_{1}(x)<1$. It follows easily that $N_{1}(x) \leqq N_{2}(x)$ for all $x$ in $X$ and hence by (a) that $N_{1} \in \mathfrak{R}$. This completes the proof of the theorem.

Let $X$ be a convex topological linear space and let $P$ be a pseudo-norm set in $X^{*}$. It follows from one of the remarks in the latter part of $\$ 1$ that if $N_{1}$ and $N_{2}$ are pseudo-norms having $P$ as pseudo-norm set then there exist positive constants $\lambda$ and $\mu$ such that $\lambda N_{1}(x) \leqq N_{2}(x) \leqq \mu N_{1}(x)$ for all $x$ in $X$. Hence it is clear from the argument given in the proof of (a) of Theorem 2 that $N_{1}$ and $N_{2}$ are either both continuous or both discontinuous. Accordingly we make: 
Definition 1. If $X$ is a convex topological linear space and $P$ is a pseudonorm set in $X^{*}$, one, and hence all, of whose generating pseudo-norms are continuous, we shall call $P$ a continuity pseudo-norm set.

Theorem 1 now tells us that there is a natural one-to-one correspondence between the convex topologies in a linear space $X$ and certain families of pseudo-norm sets and it is not difficult to deduce from Theorem 2 just what these families are. In fact let $\Im$ be such a family of continuity pseudo-norm sets. It follows from (a) under Theorem 2 that: (a') If $P_{1} \in \Im$ and $P_{2}$ is a pseudo-norm set with $P_{2} \subseteq P_{1}$ then $P_{2} \in \Im$. Furthermore it is proved in Theorem VII-5 of [9] that if $P_{1}$ and $P_{2}$ are pseudo-norm sets then $P_{1} \dot{+} P_{2}$ is a pseudo-norm set. It follows from this together with $(b)$ and $\left(a^{\prime}\right)$ that: $\left(b^{\prime}\right)$ If $P_{1}$ and $P_{2}$ are in $\Im$ then $P_{1} \dot{+} P_{2}$ is in $\Im$. Finally it is an easy consequence of the Hahn-Banach extension theorem that the intersection of the null spaces of the members of a pseudo-norm set is precisely the common null space of its generating pseudo-norms. We thus see that a family of pseudo-norms has property (c) if and only if the linear unions of their pseudo-norm sets is total. Hence $\Im$ has the property: $\left(c^{\prime}\right)$ The linear union of the members of $\Im$ is a total subspace of $X^{*}$. It follows from what we have said and the theorem of [9] to the effect that the intersection of two pseudo-norm sets is again a pseudonorm set that the set of all pseudo-norm sets in the $X^{*}$ of a linear space $X$ forms a lattice under partial ordering by inclusion. A subset $\Im$ with properties $\left(a^{\prime}\right)$ and $\left(b^{\prime}\right)$ is what is called an ideal in lattice theory. Hence we make:

Definition 2. A family of pseudo-norm sets satisfying conditions $\left(a^{\prime}\right)$, $\left(b^{\prime}\right)$, and $\left(c^{\prime}\right)$ above will be called a total ideal of pseudo-norm sets.

We may now state:

Theorem 3. Let $X$ be a linear space. Then for each convex topology in $X$ the family of continuity pseudo-norm sets is a total ideal. Conversely, given a total ideal $\Im$ of pseudo-norm sets in $X^{*}$, there is a unique convex topology in $X$ the continuity pseudo-norm sets of which are precisely the members of $\Im$.

Proof. The first part of this theorem has just been proved. The converse is an easy consequence of Theorem 2 and now familiar arguments and will be left to the reader.

If $l$ is a linear functional on a convex topological linear space $X$ it is clear that $l(x)$ is a continuous function of $x$ if and only if this is the case for $|l(x)|$. It is also clear that $|l(x)|$ as a function of $x$ is a pseudo-norm. It follows at once from Theorem 3 that the continuous linear functionals on $X$ are precisely the members of the linear union $L$ of the continuity pseudo-norm sets in $X^{*}$. Since every one-dimensional subspace of $L$ is a continuity pseudo-norm set (for the continuous pseudo-norm $|l(x)|$ where $l$ is a nonzero member of the pseudo-norm set) it follows that every finite-dimensional subspace of $L$ is a continuity pseudo-norm set. Combining these observations with Theorem 3 we conclude the truth of: 
TheOREM 4. Let $X$ be a convex topological linear space and let $L$ be the set of all continuous linear functionals defined on $X$. Then the linear system $X_{L}$ is regular, every continuity pseudo-norm set is contained in $L$ and every finitedimensional subspace of $L$ is a continuity pseudo-norm set. Conversely let $X_{L}$ be any regular linear system and let $\Im$ be any ideal of pseudo-norm sets contained in $L$ which includes every finite-dimensional subspace of $L$. Then there is a unique convex topology in $X$ whose ideal of continuity pseudo-norm sets is $\Im$. The continuous linear functionals on the resulting convex topological linear space are precisely the members of $L$.

Definition 3. If $X$ is a convex topological linear space and $L$ is the set of continuous linear functionals defined on $X$ we shall call $X_{L}$ the linear system associated with or belonging to the convex topological linear space.

Definition 4. If $X_{L}$ is a linear system and $\Im$ is a total ideal of pseudonorm sets such that (1) every finite-dimensional subspace of $L$ is a member of $\Im$ and (2) every member of $\Im$ is a subspace of $L$ we shall say that $\Im$ is an ideal associated with or belonging to the system.

Since in general there are many ideals belonging to a given regular linear system and always at least one (the ideal of all finite-dimensional subspaces of $L$ ), Theorem 4 tells us that there is a natural many-to-one correspondence between convex topological linear spaces and regular linear systems. Furthermore it tells us that there is a natural one-to-one correspondence between the ideals belonging to a regular linear system and the convex topological linear spaces to which the system belongs.

It is clear that if two convex topologies are introduced into the same linear space and if the ideal of continuity pseudo-norm sets of the first contains that of the second then every open set of the first is an open set of the second; that is, the first topology is stronger than the second and the second is weaker than the first. In particular it is clear that if $X_{L}$ is a regular linear system then the convex topology associated with the ideal of all finite-dimensional subspaces of $L$ is weaker than any other convex topology associated with $X_{L}$ and that the ideal of all ( $\left.{ }^{3}\right)$ pseudo-norm sets contained in $L$ is associated with a convex topology that is stronger than any other. Thus we may state:

THEOREM 5. If $X_{L}$ is a regular linear system, then among the convex topologies associated with $X_{L}$ there is both a weakest and a strongest.

This suggests the following definition.

Definition 5. A relatively weak (relatively strong) convex topological linear space is a convex topological linear space which has a weaker (stronger) topology than any other such space with the same linear system.

(3) That this is indeed an ideal follows at once from the fact noted above that the linear union of two pseudo-norm sets is again a pseudo-norm set. 
It is clear that a normed linear space in its norm topology is relatively strong and in its weak topology relatively weak. In case $L$ contains no infinitedimensional pseudo-norm sets, if for example $L$ is $\boldsymbol{\aleph}_{0}$-dimensional, then the relatively weak and strong topologies for $X$ associated with $X_{L}$ coincide. If however there exists at least one infinite-dimensional pseudo-norm set in $L$ then there are infinitely many convex topologies associated with $X_{L}$ which are neither relatively weak nor relatively strong. In fact we may prove the following theorem.

THEOREM 6. If $X$ is a non relatively weak convex topological linear space then there exists a second non relatively weak convex topology in $X$ which is properly weaker than the first and has the same linear system as the first.

Proof. Let $P_{1}$ be an infinite-dimensional continuity pseudo-norm set for the first topology. Let $X_{1}$ be the intersection of the null spaces of the members of $P_{1}$ and let $X_{2}$ be a complement of $X_{1}$ (that is, a not necessarily closed subspace of $X$ such that $X_{1}+X_{2}=X$ and $X_{1} \cap X_{2}=0$ ). Then $P_{1}$ as on $X_{2}$ is total and hence a norm set. Choose a subspace of $X_{2}$ closed with respect to the norms defining $P_{1}$ and having an infinite-dimensional quotient space. That this can always be done follows, for example, from the argument given on page 174 of [9]. Let $P_{2}$ be the set of all members of $P_{1}$ which vanish on this subspace. Then $P_{2}$ is an infinite-dimensional pseudo-norm set whose deficiency in $P_{1}$ is infinite. Finally consider the set $\Im$ of all pseudo-norm sets contained in pseudo-norm sets of the form $P_{2}+F$ where $F$ is a finite-dimensional subspace of $L$. It is clear that $\Im$ is an ideal associated with $X_{L}$ and that it does not contain $P_{1}$ but does contain infinite-dimensional pseudo-norm sets. Thus it defines a convex topology with the desired properties.

3. Boundedness and continuity of transformations. Kolmogoroff [8] calls a subset $A$ of a topological linear space.bounded if whenever $x_{1}, x_{2}, \cdots$ is a sequence of members of $A$ and $\gamma_{1}, \gamma_{2}, \ldots$ a sequence of real numbers converging to zero then $\gamma_{n} x_{n}$ converges to zero with respect to the topology. On the other hand von Neumann [12] calls such a subset $A$ bounded if and only if for every neighborhood of the origin $O$ in the space there exists a real number $\mu$ such that $A \subseteq \mu O$. Since Hyers [6] has proved the equivalence of these definitions we may speak without ambiguity of the bounded subsets of a topological linear space. We now prove a theorem making connections with the theory of linear systems.

TheOREM 7(4). Let $X$ be a convex topological linear space and let $X_{L}$ be its linear system. Then a subset $A$ of $X$ is bounded if and only if it is bounded as a subset of the linear system $X_{L}$; that is, if and only if, for each $l$ in $L$, l.u.b. $x \in A|l(x)|<\infty$.

Proof. The "only if" half of the theorem is obvious. Suppose conversely

(`) Cf. Smulian [14, Hilfsatz 1]. 
that l.u.b. $x \in A|l(x)|<\infty$ for all $l$ in $L$. Let $O$ be any neighborhood of the origin in $X$. Let $N$ be a pseudo-norm such that if $N(x)<1$ then $x \in O$. Now a fortiori l.u.b. ${ }_{2 \in A}|l(x)|<\infty$ for all $l$ in the pseudo-norm set of $N$. If we observe that $N$ is a norm on a complement of its null space it is easy to see that Theorème 6 on page 80 of [2] applies so that we may conclude the existence of a positive constant $\mu$ such that $N(x)<\mu$ for all $x$ in $A$. It follows that $A \subseteq \mu O$. Thus $A$ is bounded.

It is an immediate consequence of Theorem 7 that two convex topologies in $X$ generate the same bounded sets if and only if their families of continuous linear functionals have identical bounded closures. Thus it is clear that not only are there in general many convex topologies with the same continuous linear functionals but also many convex topologies with different continuous linear functionals and the same bounded sets. Examining this situation a little more closely we can gain a certain amount of insight into a problem discussed by Wehausen [18]; namely, that of the relationship between continuous linear transformations and additive transformations which take bounded sets into bounded sets. We begin by proving a lemma showing that we need only deal with linear transformations.

Lemma. If $T$ is an additive transformation from one topological linear space $X_{1}$ into another $X_{2}$ which takes bounded sets into bounded sets then $T$ is linear.

Proof. Let $x$ be an arbitrary nonzero member of $X$. If $r$ is a rational number it follows from the additivity of $T$ that $T(r x)=r T(x)$. Let $\lambda$ be any nonrational real number and let $r_{1}, r_{2}, \cdots$ be a sequence of rational numbers converging to $\lambda$. For each $n=1,2, \cdots$ let $s_{n}$ be a rational number such that $\left|\lambda-r_{n}\right| \leqq s_{n} \leqq 2\left|\lambda-r_{n}\right|$. Then $T(\lambda x)-\lambda T(x)=\left(T(\lambda x)-T\left(r_{n} x\right)\right)$ $+\left(r_{n} T(x)-\lambda T(x)\right)=s_{n}\left(T\left(\left(\left(\lambda-r_{n}\right) / s_{n}\right) x\right)\right)+\left(r_{n}-\lambda\right) T(x)$. Now $\left\{\left(\left(\lambda-r_{n}\right) / s_{n}\right) x\right\}$ is clearly a bounded sequence of members of $X_{1}$. By hypothesis then $\left.\left\{T\left(\left(\lambda-r_{n}\right) / s_{n}\right) x\right)\right\}$ is a bounded sequence of members of $X_{L}$. Hence since $s_{n}$ converges to zero $s_{n} T\left(\left(\left(\lambda-r_{n}\right) / s_{n}\right) x\right)$ must converge to zero in the topology of $X_{2}$. Since finite sets are bounded $\left(r_{n}-\lambda\right) T(x)$ also converges to zero. Thus $T(\lambda x)=\lambda T(x)$ and the lemma is proved.

Let $X$ be a linear space and let $\Im$ be any ideal of pseudo-norm sets in $X^{*}$ total or not. Let $M$ be the intersection of the null spaces of these pseudonorm sets. It is clear that the natural isomorphism between $(X / M)^{*}$ and the linear subspace $M^{\prime}$ of members of $X^{*}$ vanishing on $M$ carries each pseudonorm set whose members vanish throughout $M$ into a pseudo-norm set in $(X / M)^{*}$ and carries $\Im$ into a total ideal in $(X / M)^{*}$. Hence it follows from Theorem 4 that there is a natural one-to-one correspondence between the (not necessarily total) ideals of pseudo-norm sets in $X^{*}$ and the convex topological linear spaces defined on the quotient spaces $X / M$.

Now let $T$ be any linear transformation from $X$ onto a convex topological linear space $Y$. If $M$ is the null space of $T$ then $T$ sets up an isomorphism 
between $X / M$ and $Y$ as linear spaces. Hence in an obvious and trivial manner a convex topology may be introduced into $X / M$ in such a way that $T$ sets up an isomorphism between $Y$ and $X / M$ as topological linear spaces. Thus if we define two linear transformations to be equivalent whenever there is an isomorphism (as topological linear spaces) $U$ between their range spaces such that $U\left(T_{1}(x)\right)=T_{2}(x)$ for all $x$ in $X$, then each linear transformation $T$ from $X$ onto another convex topological linear space is equivalent to the natural transformation of $X$ onto one of its quotient spaces where this quotient space has been endowed with a suitable convex topology.

Combining the observations of the last two paragraphs we see that there is a natural one-to-one correspondence between the classes of equivalent transformations of $X$ onto topological linear spaces and the ideals of pseudonorm sets in $X^{*}$. In other words, we can survey all possible types of linear transformations from one convex topological linear space into another by considering the ordered pairs of ideals of pseudo-norm sets, the first of which is total, in the $X^{* \prime}$ 's of the various dimensional $X$ 's. Because of the order preserving nature of the correspondence between ideals and convex topologies it is clear that such a transformation is continuous if and only if the second ideal is a subset of the first and, because of Theorem 7, that such a transformation is bounded if and only if the bounded closure of the linear union of the members of the second ideal is a subset of that of the first.

It is at once clear that any continuous linear transformation carries bounded sets into bounded sets and exactly when and why the converse is not true. In fact from our point of view the converse can fail to be true in two different ways. Let $X$ be a convex topological linear space, let $X_{L}$ be the associated linear system, and let $\Im$ be the ideal of continuity pseudonorm sets. Let $\Im_{1}$ be any ideal of pseudo-norm sets in $X^{*}$ and let $L_{1}$ be the linear union of its members. If $L$ is not boundedly closed then $\Im_{1}$ may be chosen so that while the bounded closure of $L_{1}$ is contained in that of $L$ it is not true that $L_{1}$ is contained in $L$ and hence not true that $\Im_{1} \subseteq \Im_{2}$. In other words, the corresponding linear transformations will carry bounded sets into bounded sets but be discontinuous. Even if $L$ is boundedly closed, if $\Im$ fails to contain some pseudo-norm set contained in $L$ then by choosing $\Im_{1}$ as the set of all pseudo-norm sets contained in $L$ we again get a pair of ideals corresponding to a class of 'discontinuous transformations carrying bounded sets into bounded sets. On the other hand these are the only things that can happen. Suppose that $X$ is relatively strong and that $X_{L}$ is boundedly closed. If $\Im_{1}$ corresponds to a class of transformations which carry bounded sets into bounded sets so that the bounded closure of $L_{1}$ is contained in that of $L$ then, since $L$ is boundedly closed, $L_{1} \subseteq L$. Hence every member of $\Im_{1}$ is a subspace of $L$ and, since $X$ is relatively strong, is in $\Im$. Thus $\Im_{1}$ corresponds to a class of continuous transformations. This paragraph may be summarized by the following theorem. 
Theorem 8. Let $X$ be a convex topological linear space. Then the following two statements about $X$ are equivalent.

(a) Every additive (linear) transformation from $X$ into another convex topological linear space which takes bounded sets into bounded sets is continuous.

(b) $X$ is relatively strong and has a boundedly closed linear system.

Another obvious consequence of the preceding considerations generalizes Wehausen's Theorem 18.

THEOREM 9. If $X_{1}$ and $X_{2}$ are relatively weak convex topological linear spaces with boundedly closed linear systems then any additive (linear) transformation from $X_{1}$ into $X_{2}$ which takes bounded sets into bounded sets is continuous.

4. Metrizability. In this section we study conditions on the linear system and relative strength of a convex topological linear space under which it is metrizable as a topological space. We begin by observing that the proof of Theorem VII-6 on page 199 of [9] also yields a proof of the following lemma.

Lemma. Let $X$ be a linear space and let $\dot{P}_{1}, P_{2}, \ldots$ be an arbitrary sequence of pseudo-norm sets in $X^{*}$. Then if $P$ is a pseudo-norm set contained in $P_{1} \dot{+} P_{2} \dot{+} \cdots$ there exists $n=1,2, \cdots$ such that $P \subseteq P_{1} \dot{+} P_{2} \dot{+} \cdots+P_{n}$.

As an easy consequence of this we may prove:

Theorem 10. Let $X$ be a convex topological linear space and let $X_{L}$ be its linear system. Then $X$ is metrizable if and only if it is relatively strong and $L$ is the union of an ascending countable sequence of pseudo-norm sets.

Proof. Suppose that $X$ is metrizable. Then there exists a countable complete system of neighborhoods of the origin and hence a countable sequence $N_{1}, N_{2}, \cdots$ of continuous pseudo-norms such that every other continuous pseudo-norm is bounded by one of them. Clearly we may choose these so that their pseudo-norm sets form an ascending sequence, $P_{1} \subseteq P_{2} \subseteq \ldots$. Since every continuity pseudo-norm set is contained in one of the $P_{i}$ it follows that $L=P_{1}+P_{2}+\cdots$. Our lemma now implies that every pseudo-norm set contained in $L$ is contained in some $P_{i}$ and hence is a continuity pseudo-norm set. In other words, $X$ is relatively strong. Conversely let $X$ be relatively strong and let $L$ be the union of an ascending countable sequence of pseudonorm sets, $P_{1} \subseteq P_{2} \subseteq \cdots$. For each $P_{i}$ let $N_{i}$ be an associated pseudo-norm. If $O$ is any open set containing the origin and $N$ is a continuous pseudo-norm such that $N(x)<1$ implies that $x$ is in $O$ it follows upon applying the lemma to the pseudo-norm set of $N$ that, for some $n=1,2, \cdots$ and some $\mu>0$, $N(x) \leqq \mu N_{n}(x)$ for all $x$ in $X$ so that the set where $N_{n}(x)<1 / \mu$ is included in $O$. Thus the open sets defined by the inequalities $N_{i}(x)<1 / j$ for $i, j=1,2, \ldots$. form a complete system of neighborhoods of the origin. It follows at once from the Birkhoff-Kakutani group metrizability criterion that $X$ is metriza- 
ble. As a matter of fact, as the reader may show directly without difficulty, $\rho(x, y)=\sum_{n=1}^{\infty} N_{n}(x-y) / 2^{n}\left(1+N_{n}(x-y)\right)$ is a metric.

Since the linear union of countably many pseudo-norm sets is always boundedly closed [9, Theorem V-13] we may state the following corollary.

COROLLARY. The linear system of a metrizable convex topological linear space is always boundedly closed.

Theorem 10 tells us that there is a natural one-to-one correspondence between the metrizable convex topologies in a linear space $X$ and the total subspaces of $X^{*}$ which are the unions of ascending sequences of pseudo-norm sets. It follows from the lemma at the beginning of this section that such a union is a norm set if and only if all of the terms past a certain one are identical. Thus the metrizable but non-normable convex topologies correspond to the stric " increasing sequences. It follows from the general theory of pseudonorm sets as developed in [9] that such sequences exist in great variety whenever $X$ is infinite-dimensional.

An interesting special case occurs when all the pseudo-norm sets are finitedimensional. In this case $L$ is not more than $\aleph_{0}$-dimensional and the space itself is relatively weak as well as relatively strong. Conversely if $X_{L}$ is an arbitrary regular linear system in which $L$ is not more than $\aleph_{0}$-dimensional, it follows from the fact that an infinite-dimensional pseudo-norm set must be at least $C$-dimensional [9, Theorem I-1] that the only pseudo-norm sets in $L$ are finite-dimensional and that $L$ is the union of a countable ascending sequence of them. Thus there is a unique convex topology associated with $X_{L}$ and it is metrizable. The most familiar example of such a space is Banach's $[2, \mathrm{p} .10]$ space $(s)$ consisting of all sequences of real numbers where

$$
\rho\left(x_{1}, x_{2}, \cdots ; y_{1}, y_{2}, \cdots\right)=\sum_{n=1}^{\infty}\left|x_{n}-y_{n}\right| / 2^{n}\left(1+\left|x_{n}-y_{n}\right|\right) .
$$

It is readily verified that the topology defined by this metric is the unique convex topology whose continuous linear functionals are the finite linear combinations of the functionals $f_{i}$ where, for each $i=1,2, \cdots, f_{i}\left(x_{1}, x_{2}, \cdots\right)=x_{i}$. We show now that the space $(s)$ is a universal space of this character.

Theorem 11. A relatively weak convex topological linear space is metrizable if and only if it is isomorphic (as a topological linear space) to a subspace of the space (s) of Banach. It is normable if and only if it is finite-dimensional.

Proof. The "if" parts of both statements are obvious. The "only if" part of the second statement is an immediate consequence of the fact that a strictly ascending sequence of pseudo-norm sets never has a norm set for its sum. To prove the "only if" part of the first statement let $X_{L}$ be the linear system of a metrizable relatively weak convex topological linear space. Then $L$ is $\aleph_{0}$-dimensional and admits a Hamel basis $l_{1}, l_{2}, \ldots$ For each $x$ in $X$ 
let $F(x)=l_{1}(x), l_{2}(x), \cdots$. Then $F$ sets up a one-to-one linear map of $X$ into part of $(s)$ in such a way that $l_{i}(x)=f_{i}(F(x))$ for all $x$ in $X$. Thus since the topology of $X$ is defined by the $l_{i}$ and that of $(s)$ by the $f_{i}$ it is easily seen that $F$ is a homeomorphism. This completes the proof of the theorem.

We close this section with an example of a metrizable non-normable topological linear space of interest in function theory. Let $R$ be a bounded open set in the complex $z$-plane. Let $\mathfrak{F}$ be the linear space of all single-valued functions defined and analytic throughout $R$. We introduce a topology in $\mathfrak{F}$ as follows. For each $n=1,2, \cdots$ let $C_{n}$ be the set of all points in $R$ whose distance from the boundary of $R$ is at least $1 / n$. Then $C_{n}$ is a closed subset of $R$. Let $N_{n}(f)=1$.u.b.s $\in C_{n}|f(z)|$ for each $f$ in $\mathfrak{F}$ and each $n=1,2, \cdots$. Clearly $N_{n}$ is not only a pseudo-norm but, for $n$ large enough so that $C_{n}$ is not empty, also a norm. Let $P_{n}$ be the norm set associated with $N_{n}$. Let $L=P_{1}+P_{2}+\cdots$ and introduce in $\mathfrak{F}$ the relatively strong convex topology associated with $\mathfrak{F}_{L}$. It follows easily from well known theorems in function theory that uniform boundedness on $C_{n}$ does not imply uniform boundedness on $C_{n+1}$. Accordingly it is clear that the sequence of pseudo-norm sets $P_{1}, P_{2}, \ldots$ is strictly ascending so that the topology we obtain is metrizable but not normable. It is readily verified that convergence in this topology is equivalent to uniform convergence on every closed subset of $R$.

5. Completeness. A topological linear space, being a topological group, has a natural uniform structure. (See Weil [19] for a discussion of this notion.) Hence one may speak of its totally' bounded subsets, its Cauchy directed systems of elements, and of whether or not it is complete. Completeness with respect to general Cauchy directed systems turns out to be rather rare and various authors have introduced several weaker notions which we shall now formulate.

Definition 6. A topological linear space is $C_{4}$ complete if it is complete as a uniform structure with respect to the convergence of directed systems. It is $C_{3}$ complete if every closed and bounded subset is $C_{4}$ complete. It is $C_{2}$ complete if every closed and totally bounded subset is $C_{4}$ complete. It is $C_{1}$ complete if it is complete as a uniform structure with respect to the convergence of sequences. It is $T_{2}$ complete if every closed and totally bounded subset is bicompact. It is $T_{1}$ complete if every closed and totally bounded subset is sequentially compact.

Completeness in topological linear spaces was apparently first discussed by von Neumann [12] who introduced what we have called $C_{1}$ and $T_{1}$ completeness. $C_{4}$ completeness was introduced by G. Birkhoff [4], $C_{3}$ completeness by Taylor $[15,16]$, and $C_{2}$ completeness by Šmulian [14]. It is readily verified that for $i=2,3$, or $4, C_{i}$ completeness implies $C_{i-1}$ completeness and that $T_{2}$ completeness implies $C_{2}$ completeness. That $C_{2}$ completeness implies $T_{2}$ completeness follows from the generalization to uniform structures of the well known theorem that a complete totally bounded metric space is bicom- 
pact [19, Théorème 7] or from a trivial modification of G. Birkhoff's [4] proof that $C_{4}$ completeness implies $T_{2}$ completeness. Finally von Neumann [12] has proved that $T_{1}$ completeness implies $C_{1}$ completeness. The relationship between the various notions of completeness may thus be represented schematically as follows:

$$
C_{4} \rightarrow C_{3} \rightarrow C_{2}=T_{2} \rightarrow T_{1} \rightarrow C_{1} \text {. }
$$

In discussing compieteness in convex spaces our principal tool will be a sort of partial completion, the elements of which depend only upon the linear system of the space in question. Let $X_{L}$ be an arbitrary regular linear system and in the usual manner regard $X$ as a subspace of $L^{*}$. Consider the family $\mathfrak{A}$ of all subsets $A$ of $L$ which are uniformly bounded as members of the conjugate system $L_{X}$. Let $X^{-} \supseteq X$ be the set of all $f$ in $L^{*}$ for which l.u.b. $\iota \in A|f(l)|$ $<\infty$ for all $A$ in $\mathfrak{A}$. Next let $N$ be any pseudo-norm defined in $X$ whose pseudo-norm set is contained in $L$. Following the procedure familiar for norms we define $N(l)=1$.u.b. $N(x)=1|l(x)|$ for each $l$ in $L$. Finally we define $N^{-}(f)$ $=$ l.u.b. $N(l)=1|f(l)|$ for all $f$ in $X^{-}$.

LEMma 1. $N^{-}(f)$ is finite for all $f$ in $X^{-}$and is a pseudo-norm as a function of $f$. Furthermore $N(x)=N^{-}(x)$ for all $x$ in $X$. Finally if $N_{1}(x) \leqq N_{2}(x)$ for all $x$ in $X$ then $N_{1}^{-}(f) \leqq N_{2}^{-}(f)$ for all $f$ in $X^{-}$.

Proof. Let $B$ be any bounded subset of $X_{L}$. Then in particular $l(B)$ is a bounded set of real numbers for each $l$ in the pseudo-norm set $P_{N}$ of $N$. Hence $N(B)$ is a bounded set of real numbers $\left(^{5}\right)$. But $|l(x)| \leqq N(l) N(x)$ for all $l$ in $P_{N}$ and all $x$ in $X$. Thus l.u.b. $x \in B, N(l)=1,|l(x)|<\infty$ so that the set of all $l$ with $N(l)=1$ is uniformly bounded. Hence $N^{-}$is finite on $X^{-}$. That $N^{-}$is a pseudo-norm follows from the easily proved general theorem that the supremum of any pointwise bounded family of pseudo-norms is again a pseudonorm. That $N(x)=N^{-}(x)$ for $x$ in $X$ follows readily from its well known truth in the case where $N$ is a norm. Finally the last statement is an obvious consequence of the definitions concerned.

Now let $t$ be any convex topology associated with $X_{L}$ and let $\mathfrak{M}$ be the class of all pseudo-norms $M$ defined on $X^{-}$such that, for some continuous pseudo-norm $N$ defined on $X, M(f) \leqq N^{-}(f)$ for all $f$ in $X^{-}$.

LEMmA 2. There is a unique convex topology $t^{-}$in $X^{-}$whose continuous pseudo-norms are precisely the members of $\mathfrak{M}$. This topology coincides on $X$ with $t$. If $t_{1}$ and $t_{2}$ are two convex topologies associated with $X_{L}$ and $t_{1}$ is stronger than $t_{2}$ then $t_{1}^{-}$is stronger than $t_{2}^{-}$.

Proof. If $N_{1}$ and $N_{2}$ are pseudo-norms on $X$ continuous with respect to $t$ then $N_{1}+N_{2}$ is again such and by the preceding lemma $\left(N_{1}+N_{2}\right)^{-}(f)$ $\geqq \max \left(N_{\overline{1}}(f), N_{2}^{-}(f)\right)$ for all $f$ in $X$. If $f \neq 0$ choose $l$ in $L$ with $f(l) \neq 0$ and a con-

(5) Cf. proof of Theorem 7. 
tinuous pseudo-norm $N$ containing $l$ in its pseudo-norm set. Then $N^{-}(f) \neq 0$. It follows from these remarks and the mode of definition of $\mathfrak{M}$ that $\mathfrak{M}$ satisfies the conditions (a), (b), and (c) of Theorem 2. The truth of the first statement of this lemma now follows from Theorem 2. The truth of the second statement follows at once from Theorem 1 and the truth of the third follows from the fact (obvious from the proof of Theorem 1) that one convex topology is stronger than another if and only if it has more continuous pseudo-norms.

Lemma 3. With respect to the topology $t^{-}$every bounded Cauchy directed system $\left\{x_{\alpha}\right\}$ in $X$ has a limit in $X^{-}$.

Proof. Let $l$ be an arbitrary member of $L$. Since $l$ is continuous and linear it is readily verified that $\left\{l\left(x_{\alpha}\right)\right\}$ is a Cauchy directed system of real numbers and hence has a limit $f(l)$. The number $f(l)$ as a function of $l$ is clearly a member of $L^{*}$. Let $A$ be any uniformly bounded subset of $L_{X}$. Then

$$
\text { l.u.b. } \in_{A}\left|l\left(x_{\alpha}\right)\right|=c<\infty \text {. }
$$

Hence $|f(l)| \leqq c$ for all $l$ in $A$. Thus $f \in X^{-}$. To show that $x_{\alpha} \rightarrow f$ it is manifestly sufficient to show that for each continuous pseudo-norm $N$ on $X$ we have $N^{-}\left(x_{\alpha}-f\right)$ tending to zero. Given $\epsilon>0$, choose $\gamma$ so that if $\alpha>\gamma$ and $\beta>\gamma$ then $N^{-}\left(x_{\alpha}-x_{\beta}\right)<\epsilon / 2$. Choose any $\alpha>\gamma$. Since for each $l$ in $L$ we have $\left|l\left(x_{\alpha}\right)-f(l)\right|$ $=\lim _{\beta}\left|l\left(x_{\alpha}\right)-l\left(x_{\beta}\right)\right|$ and for each $\beta$ we have $\left|l\left(x_{\alpha}\right)-l\left(x_{\beta}\right)\right| \leqq N(l) N\left(x_{\alpha}-x_{\beta}\right)$ it follows that $\left|l\left(x_{\alpha}\right)-f(l)\right| \leqq N(l)(\epsilon / 2) \leqq \epsilon / 2$ if $N(l) \leqq 1$. Thus since $N^{-}\left(x_{\alpha}-f\right)$ $=1$.u.b.N(l)=1 $\left|l\left(x_{\alpha}\right)-f(l)\right|$ it follows that $N^{-}\left(x_{\alpha}-f\right) \leqq \epsilon / 2<\epsilon$. Thus $N^{-}\left(x_{\alpha}-f\right)$ $\rightarrow 0$ and the lemma is proved.

As a first consequence of these considerations we prove the following theorem.

Theorem 12. If $i$ is 1,2 , or 3 and $X$ is a $C_{i}$ complete convex topological linear space then under any "relatively stronger" convex topology $X$ is again $C_{i}$ complete.

Proof. We shall consider only the case $i=2$. The other cases are similar and slightly easier. Let $t_{1}$ be the weaker and $t_{2}$ the stronger of the two topologies. Let $X_{L}$ be their common linear system and let $X^{-}$be the subspace of $L^{*}$ introduced above. Extend $t_{1}$ and $t_{2}$ to topologies $t_{1}^{-}$and $t_{2}^{-}$defined in $X^{-}$as described in Lemma 2. Let $\left\{x_{\alpha}\right\}$ be a directed system of elements of $X$, Cauchy and totally bounded with respect to $t_{2}$. By Lemma 3 the system $\left\{x_{\alpha}\right\}$ has a limit $f$ in $X^{-}$with respect to $t_{2}^{-}$. Since, by Lemma $2, t_{2}^{-}$is stronger than $t_{1}^{-}$it follows that $f$ is also a limit of $\left\{x_{\alpha}\right\}$ with respect to $t_{1}^{-}$. Hence $\left\{x_{\alpha}\right\}$ is Cauchy with respect to $t_{1}$. Furthermore since $t_{1}$ is weaker than $t_{2},\left\{x_{\alpha}\right\}$ is totally bounded with respect to $t_{1}$. But $X$ is $C_{2}$ complete in the $t_{1}$ topology. Since limits of directed systems are unique it follows that $f$ is in $X$. Thus $X$ is $C_{2}$ complete in the $t_{2}$ topology and the theorem is proved.

Similar considerations give us the following theorem. 
TheOREM 13. If $X$ is a convex topological linear space which is $C_{1}$ complete (and hence if $X$ is complete in any sense) then the linear system $X_{L}$ associated with $X$ is a complete linear system.

Proof. Let $x_{1}, x_{2}, \cdots$ be any sequence of elements of $x$ which converges as a sequence of elements of the linear system $X_{L}$ to a member $f$ of $L^{*}$. Then by the definition of convergence there exists a sequence $\gamma_{1}, \gamma_{2}, \cdots$ of real numbers, converging to zero, such that $\left\{\left(l\left(x_{n}\right)-f(l)\right) / \gamma_{n}\right\}$ is bounded for each $l$ in $L$. It follows in particular that $l\left(x_{n}\right) \rightarrow f(l)$ for each $l$ and that $\left\{l\left(x_{n}-x_{m}\right) /\left(\gamma_{n}+\gamma_{m}\right)\right\}$ is bounded in $m$ and $n$ for each $l$. Let $l_{1}, l_{2}, \ldots$ be any uniformly bounded sequence of elements of $L_{x}$. Then $\left|l_{k}\left(x_{n}\right)\right| \leqq \mu$ for all $k=1,2, \ldots$ and $n=1,2, \ldots$ so that $\left|f\left(l_{k}\right)\right| \leqq \mu$ for all $k=1,2, \ldots$ where $\mu$ is some suitable positive real number. Hence $f$ is a member of $X^{-}$. Let $N$ be any continuous pseudo-norm defined on $X$. Then $N^{-}\left(x_{n}-f\right)=1$.u.b.N(l)-1 $\left|l\left(x_{n}\right)-f(l)\right|$ $=$ l.u.b.N(l)-1 $\lim _{m+\infty}\left|l\left(x_{n}-x_{m}\right)\right|=$ l.u.b.N(l)-1 $\lim _{m \rightarrow \infty}\left(\gamma_{n}+\gamma_{m}\right) l\left(\left(x_{n}-x_{m}\right) /\left(\gamma_{n}+\gamma_{m}\right)\right)$. But since the set of all $l$ with $N(l)=1$ is uniformly bounded and the set of all $\left\{\left(x_{n}-x_{m}\right) /\left(\gamma_{n}+\gamma_{m}\right)\right\}$ for $n, m=1,2, \ldots$ is bounded it follows that there exists a constant $\mu>0$ with $\left|l\left(\left(x_{n}-x_{m}\right) /\left(\gamma_{n}+\gamma_{m}\right)\right)\right| \leqq \mu$. Thus $N^{-}\left(x_{n}-f\right)$ $\leqq$ l.u.b. $(l)-1$ lim $\sup _{m \rightarrow \infty}\left(\gamma_{n}+\gamma_{m}\right) \mu \leqq \gamma_{n} \mu$. Hence $N^{-}\left(x_{n}-f\right) \rightarrow 0$. It follows then from the connection between the $N^{-}$'s and the topology of $X^{-}$that $x_{n} \rightarrow f$. But since $X$ is $C_{1}$ complete, $f$ is in $X$. Thus $X_{L}$ is a complete linear system.

We do not know whether or not it is true that every complete linear system is associated with $a$, in some sense complete, convex topological linear space. We do have, however, the following theorem.

Theorem 14. If $X_{L}$ is a complete linear system such that $X$ is a boundedly closed subspace of $L^{*}$ then every convex topology associated with $X_{L}$ is $C_{1}, C_{2}, C_{3}$, $T_{1}$, and $T_{2}$ complete.

Proof. By Theorem VI-4 of [9], $X_{L}$ is uniform. Hence since $X$ is boundedly closed as a subspace of $L^{*}$ it follows that $X^{-}=X$. The truth of the theorem now follows from Lemma 3 and the fact that $C_{3}$ completeness implies all the other kinds under consideration.

That $C_{4}$ completeness cannot be included may be seen from the following theorem which is a trivial generalization of related theorems of $G$. Birkhoff [4, page 49] and Taylor [16, Theorem 4.9].

TheORem 15. If $X$ is a relatively weak $C_{4}$ complete convex topological linear space then the linear system $X_{L}$ associated with $X$ is of the form $Y_{Y}^{*}$, that is, $X=L^{*}$.

Proof. Let $f$ be any member of $L^{*}$. For each finite-dimensional subspace $M$ of $L$ let $x_{M}$ be a member of $X$ such that $x$ as a member of $L^{*}$ coincides on $M$ with $f$. This is possible since $X$ as on $M$, being total, is the whole of $M^{*}$. If we partially order the finite subspaces of $L$ by inclusion $\left\{x_{M}\right\}$ becomes a directed system. Using the fact that the topology of $X$ is relatively weak it is 
easy to show that $\left\{x_{M}\right\}$ converges to $f$. Since $X$ is $C_{4}$ complete, $f$ must be in $X$ and the theorem follows.

The question arises as to whether or not Theorem 14 has a converse; that is, as to whether or not the linear system of a relatively weak convex topological linear space which is $C_{3}$ complete necessarily has a boundedly closed conjugate. We do not know the answer to this question. We note, however, in view of an obvious converse of Theorem 15 that an affirmative answer would imply that a linear space $X$ is always a boundedly closed subspace of $X^{* *}$ and hence, by virtue of Theorem IV-12 of [9], answer a measure theoretic question raised by Ulam [17]. Furthermore we do have the following theorem.

THEOREM 16. If $X_{L}$ is the linear system of a $T_{2}$ complete relatively weak convex topological linear space and if $X_{L}$ is relatively bounded then $X_{L}^{0}$ is boundedly closed.

Proof. By Theorem 13, $X_{L}$ is a complete linear system. Hence, since $X_{L}$ is relatively bounded, it follows from the corollary to Theorem VI-4 of [9] that $X_{L}$ is simple. Thus by Theorem $\mathrm{V}-17$ of [9] the bounded closure $\bar{L}$ of $L$ is a norm set. Let $N_{1}$ be a generating norm. For each $x$ in $X$ let $N(x)$ $=1$.u.b. $N_{1}(l)-1|l(x)|$. Then $N(x) \leqq N_{1}(x)$ so that the norm set of $N$ is contained in $L$. On the other hand, this norm set contains $L$. Hence $\bar{L}$ is also the norm set of $N$. Let $K$ be the set of all $x$ in $X$ for which $N(x) \leqq 1$. An element $x$ of $X$ is in $K$ if and only if $|l(x)| \leqq 1$ for all $l$ in $L$ such that $N(l)=1$. Hence $K$ is an intersection of sets closed in the topology of $X$ and is consequently itself closed in this topology. Obviously $K$ is bounded. But it has been shown by Šmulian $[14$, Hilfsatz 2$]$ and essentially by Taylor $[16$, Theorem 4.3$]$ that in a relatively weak convex topological linear space boundedness and total boundedness are equivalent. Thus $K$ is totally bounded. Since $K$ is closed and totally bounded and $X$ is $T_{2}$ complete it follows that $K$ is bicompact. Thus by Theorem VII-4 of [9] $K+$ is a norm set. Since $K+=X$ and norm sets are boundedly closed the theorem is proved.

Theorems 14 and 16 tell us that the relatively weak convex topological linear space associated with a regular relatively bounded linear system $X_{L}$ is $C_{3}\left(T_{2}, C_{2}\right)$ complete if and only if $X_{L}$ is complete and has a boundedly closed conjugate. We characterize such linear systems now in terms of the notion of an almost complete normed linear space [9, p. 196].

TheoRem 17. Let $X_{L}$ be a regular linear system. Then $X_{L}$ is relatively bounded, complete, and such that $X_{L}^{0}$ is boundedly closed if and only if $X_{L}^{0}$ is the linear system of an almost complete normed linear space.

Proof. If $X_{L}$ is relatively bounded and complete it follows from Theorems VI-4 and VI-2 of [9] that $X_{L}$ and $X_{L}^{0}$ are simple and uniform. Since $X_{L}^{0}$ is boundedly closed and simple it is in the linear system of a normed 
linear space [9, Theorem V-17]. Since it is uniform the normed linear space is almost complete. Conversely, if $X_{L}^{0}$ is the linear system of an almost complete normed linear space then it follows immediately that $X_{L}^{0}$ is boundedly closed and relatively bounded. Hence $X_{L}$ is relatively bounded. It remains to show that $X_{L}$ is complete. But since $X_{L}^{0}$ is uniform and boundedly closed $X^{-}=X$. Hence, by Lemma 3, every convex topological linear space associated with $X_{L}$ is $C_{3}$ complete. Thus, by Theorem $13, X_{L}$ is a complete linear system.

As is pointed out on page 195 of [9], if $X_{L}$ is the linear system of a normed linear space then the uniformly bounded subsets of $X_{L}$ are precisely the norm bounded subsets of $L$. Thus if the normed linear space is almost complete (that is, if $X_{L}$ is uniform) then it is reflexive if and only if $X_{L}^{0}$ is boundedly closed. Hence since a reflexive space is always complete it follows that a normed linear space is reflexive if and only if its linear system is complete and has a boundedly closed conjugate. Thus we obtain from the foregoing another proof of the well known theorem:

TheOREM 18. For a normed linear space $X$ the following are equivalent: (a) $X$ is reflexive, (b) $X$ is $C_{3}$ complete in its weak topology, (c) $X$ is $T_{2}$ complete in its reak topology.

6. Category. Wehausen [18, Theorems 15 and 16] has shown that a complete topological linear space need not be of the second category by proving that an infinite-dimensional normed linear space in its weak topology is necessarily of the first category. In this section we give two necessary conditions that a convex topological linear space be of the second category, each of which implies Wehausen's result. The first of these is essentially due (in another formulation) to Sirvint [13].

THEOREM 19. If $X$ is a convex topological linear space of the second category then it is relatively strong and its linear system $X_{L}$ is uniform.

Proof. Let $\left\{l_{\alpha}\right\}$ be any bounded subset of $L_{X}$. For each $x$ in $X$ let $N(x)=$ l.u.b. $\left|l_{\alpha}(x)\right|$. Then $N$ is a pseudo-norm and the set $K$ of all $x$ with $N(x) \leqq 1$ is an intersection of closed sets and hence closed. Since $K \cup 2 K$ $\cup 3 K \cup \ldots=X$ and $X$ is of the second category it follows that some $n K$ has an interior point. Hence $K$ has an interior point $\bar{x}$. Thus there exists a continuous pseudo-norm $N_{1}$ such that if $N_{1}(y)<1$ then $\bar{x}+y$ is in $K$; that is, $N(\bar{x}+y) \leqq 1$. It follows easily that there exists a positive real number $\mu$ such that $N(x) \leqq \mu N_{1}(x)$ for all $x$ in $X$. Hence, by Theorem $2, N$ is continuous. Since any pseudo-norm whose pseudo-norm set is in $L$ can be generated in this way it follows that $X$ is relatively strong. To prove the uniformity of $X_{L}$ let $x_{1}, x_{2}, \cdots$ and $l_{1}, l_{2}, \ldots$ be bounded subsets of $X_{L}$ and $L_{X}$ respectively. By the argument just presented 1.u.b.i=1,2, $\ldots\left|l_{i}(x)\right|$ is a continuous pseudonorm. Hence l.u.b.i=1,2, $\ldots\left|l_{i}\left(x_{j}\right)\right|$ is bounded with respect to $j$; that is, 1.u.b. $i, j=1,2, \ldots\left|l_{i}\left(x_{j}\right)\right|<\infty$. Thus $X_{L}$ is uniform and the theorem is proved. 
If $L$ is not "too large" a subspace of $X^{*}$ we can conclude considerably more.

THEOREM 20. If $X$ is a convex topological linear space of the second category and its linear system $X_{L}$ is almost relatively bounded then $X$ is normable as an almost complete normed linear space.

Proof. Since $X_{L}$ is almost relatively bounded we have $X=B_{1} \cup B_{2} \cup \ldots$ where each $B_{i}$ is bounded. By a theorem of von Neumann [12, Theorem 11] the closure of each $B_{i}$ is bounded. Since $X$ is of the second category at least one $B_{i}$ has an interior point. Since $X$ is convex $B_{i}$ contains a convex open set. Thus $X$ contains a bounded convex open set and hence by a well known theorem of Kolmogoroff [8] is normable. That the resulting normed linear space is almost complete follows from the uniformity part of the conclusion of Theorem 19.

7. The lattice of closed subspaces. It is an easy consequence of the HahnBanach extension theorem that the closed subspaces of the convex topological linear space are the intersections of null spaces of continuous linear functionals. Thus the closed subspaces of $X$ are precisely the closed subspaces of the linear system $X_{L}$ [9, chap. III]. It follows immediately that all of the convex topological linear spaces associated with $X_{L}$ have mutually isomorphic lattices of closed subspaces. Thus the theorem of [11] to the effect that a normed linear space is determined to within isomorphism by its lattice of closed subspaces does not extend to general convex topological linear spaces. On the other hand, since it is true that a linear system is determined to within isomorphism by its lattice of closed subspaces [9, Theorem III-3], we may state the following theorem.

THEOREM 21. If $X$ and $Y$ are relatively strong (relatively weak) convex topological linear spaces then they are isomorphic (that is, homeomorphic and algebraically isomorphic under the same mapping) if and only if their lattices of closed subspaces are isomorphic as abstract lattices.

8. The ring of continuous linear transformations. Let $X$ be a convex topological linear space and let $T$ be a linear transformation of $X$ into itself. It is easy to see that $T$ is continuous if and only if for each continuous pseudonorm $N$ on $X$ the pseudo-norm $N(T(x))$ is also continuous. Furthermore as may be verified without difficulty the pseudo-norm set of $N(T(x))$ is the set of all functionals $f$ of the form $f(x)=l(T(x))$ where $l$ is in the pseudo-norm set of $N$. In other words, $T$ is continuous if and only if whenever $M$ is a continuity pseudo-norm set then $T^{*}(M)$ is also. It follows that every continuous $T$ is a homomorphism [9, page 160] of the linear system $X_{L}$ into itself and that the converse is true if and only if the continuity pseudo-norm sets are collectively invariant under the conjugates of homomorphisms of $X_{L}$ into itself. This last condition will certainly hold if the continuity pseudo-norm 
sets are all pseudo-norm sets in $L$ or all finite-dimensional pseudo-norm sets in $L$ but, as one may show by examples, will not hold in general. One may show moreover that whenever the ring of continuous linear transformations is not equal to the ring of homomorphisms of the linear system it is not isomorphic to it. The situation for rings is then a little different than that for lattices. Some but not all of the different convex topological linear spaces associated with a given linear system will be mutually isomorphic. However, as in the lattice case, we may conclude that while the isomorphism theorem for normed linear spaces does not extend to general convex topological linear spaces we do have the following theorem.

THEOREM 22. If $X$ and $Y$ are relatively strong (relatively weak) convex topological linear spaces then they are isomorphic if and only if their rings of continuous linear transformations are isomorphic as abstract rings.

\section{BIBLIOGRAPHY}

1. P. Alexandroff and H. Hopf, Topologie, vol. 1, Berlin, 1935.

2. S. Banach, Theorie des operations linéaires, Warsaw, 1932.

3. G. Birkhoff, A note on topological groups, Compositio Math. vol. 3 (1936) pp. 427-430. 39-56.

4. - Moore-Smith convergence in general topology, Ann. of Math. vol. 38 (1937) pp.

5. G. Fichtenholz, Sur les fonctionelles linéaires continues au sens generalise, Rec. Math. (Mat. Sbornik) N. S. vol. 4 (1938) pp. 192-213.

6. D. H. Hyers, $A$ note on linear topological spaces, Bull. Amer. Math. Soc. vol. 44 (1938) pp. 76-80.

7. S. Kakutani, Ueber die Metrization der topologischen Gruppen, Proc. Imp. Acad. Tokyo vol. 12 (1936) pp. 82-84.

8. A. Kolmogoroff, Zur Normierbarkeit eines allgemeinen topologischen linearen Raumes, Studia Mathematica vol. 5 (1935) pp. 29-33.

9. G. W. Mackey, On infinite-dimensional linear spaces, Trans. Amer. Math. Soc. vol. 57 (1945) pp. 155-207.

10. - On convex topological linear spaces, Proc. Nat. Acad. Sci. U. S. A. vol. 29 (1943) pp. 315-319.

11. - On isomorphisms of normed linear spaces, Ann. of Math. vol. 43 (1942) pp. 244260.

12. J. von Neumann, On complete topological spaces, Trans. Amer. Math. Soc. vol. 37 (1935) pp. 1-20.

13. G. Sirvint, Espace de fonctionelles lineaires, C. R. (Doklady) Acad. Sci. URSS. vol. 26 (1940) pp. 123-126.

14. V. Smulian, Über lineare topologische Railme, Rec. Math. (Mat. Sbornik) N. S. vol. 7 (1940) pp. 425-448.

15. A. E. Taylor, The weak topologies of Banach spaces, Proc. Nat. Acad. Sci. U. S. A. vol. 25 (1939) pp. 438-440.

16. - The weak topologies of Banach spaces, Revista de Ciencias vol. 43 (1941) pp. 355-365, 465-474, 667-674 and vol. 44 (1942) pp. 45-63.

17. S. Ulam, Zur Masstheorie in der allgemeinen Mengenlehre, Fund. Math. vol. 16 (1930) pp. 140-150. 
18. J. V. Wehausen, Transformations in linear topological spaces, Duke Math. J. vol. 4 (1938) pp. 157-169.

19. A. Weil, Sur les espaces d structure uniforme, Actualités Scientifiques et Industrielles, No. 551, Paris, 1938.

20. H. Whitney, Tensor products of abelian groups, Duke Math. J. vol. 4 (1938) pp. 495528.

\section{HARVARD UNIVERSITY,}

Cambridge, Mass. 